\title{
Note sur l'estimation des débits de crues
}

\section{On the estimation of flood discharges}

\author{
PAR G. MORLAT

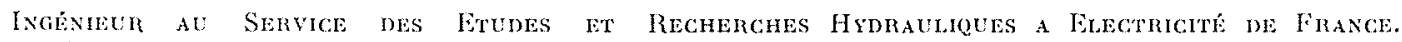 \\ English synopsis, p. 580 .
}

La question des débits de crue donne lieu aux tranaux d'une Commission spéciale de la S.H.F.

Ce travail de M. Monut donne le point de vue d'un statisticien tel cu'ti l'a exposé a la première réunion de cette Commission.

\section{SOM M A IRE}

I. - Introtuction.

Définition d'une crue. - Importance de considerations économiques dans le choix de la crue it prevoir.

II. - L'Emprot De fomules emprotques.

Nous classons, sous cette rubrique traditionnclle, les möhodes visant ì déterminer un débil de crue d'apres des renseignements très généraux : le plus souvent, ce sera l'ensemble des plus grandes crues observées dans une région, ou mêne à la surface du globe.

III. - Les MÉTHOdes sthTISTrQues.

Ces méthodes ont été appliquées presque exclusivement à l'analyse de relevés de débits concernant le cours d'ean étudié. Leur emploi a domné lieu parlois à des erreurs graves, par suite d'une comnaissance insuffisante de certaines notions statistiques élémentaires.

IV. - Les méthodes ANALyTrques.

Il s’agit ici des procédés dont l'objet essentiel est l'analyse du mécanisme de formation des débits à partir des pluies. Leur caractère analytique est en réalité plus ou moins prononcé, selon la part qui est attribuée aux pro- priétés hydrauliques du bassin. Les variantes en sont fort nombreuses.

V. - PossibILITÉ DE SYNTHÉSE PLUIES-DÉBtTs.

Un outil mathématique extrêmement puissant a pu être introduit (Le CAM), traduisant en termes précis l'essentiel des méthodes envisagées au chapitre précédent. Cet outil doit permettre de combiner les renseignements apportés par les séries pluviométriques et hydrométriques, ainsi que certaines propriètés hydrauliques du bassin.

VI. - LA NoTION dE PROBABHité EN hydrologie.

Mais pour justifier pleinement une synthèse logique des diverses méthodes d'approche, comme pour utiliser éventuellement d'autres informations, de caractère plus (qualitatif, bien que non négligeables, il esi nécessaire de revenir sur les fondements de la notion de probabilité. De nombreux spécialistes, dans le souci louable de résoudre des difficultés classiques, ont abouti à une notion for $\dot{i}$ restreinte, bien que précieuse dans des problèmes tels que le contrôle des fabrications (fréquentistes); il faut au contraire, en hydrologie, adopter les vues plus profondes exprimées par d'autres auteurs (Keynes, HalpheN).

\section{I. - INTRODUCTION}

Le présent mémoire vise, d'une part, à rendre compte de l'étal actuel du problème, tel qu'il ressort des communications présentées au Congrès des Grands Barrages de New-I)elhi -- et d'autre part à préciser les raisons profondes, et quelque peu philosophiques, de l'emploi de la notion de probabilité en hydrologie : cette notion joue en effet un rôle tout à fait primordial dans 
la détermination des crues à prévoir pour les ouvrages hydrauliques.

Des communications présentées à New-Delhi, je retiendrai seulement celles qui se rattachent assez directement à l'aspect hydrologique du problème, laissant de còté les exposés, par ailleur's fort intéressants, qui avaient trait à des aspects différents : étude des propriétés hydrauliques des déversoirs, de leur disposition, propagation des intumescences, etc.

\section{IMPORTANCE DU PROBLEME.}

L’importance du problème hydrologique a été soulignée par un grand nombre d'auteurs. Pour citer seulement M. PARDÉ, des excmples montrant « les consécquences funestes que peut avoir la sous-estimation des débits de crue $\gg$ sont bien connus. M. PardÉ nous en rappelle quelques-uns parmi les plus frappants : le désastre de l'Orba à Ortiglieto en 1935 , dû à un débit de $2.200 \mathrm{~m}^{3} / \mathrm{s}$, alors qu'on n'avait prévu que $850 \mathrm{~m}$, - celui du barrage de Fergoug (Algérie) sur l'oued Hammam, en 1927, avec une crue de l'ordre de 4.000 à $5.500 \mathrm{~m}^{3} / \mathrm{s}\left(1.500 \mathrm{~m}^{3}\right.$ prévus), et le débit de $7.350 \mathrm{~m}^{3} / \mathrm{s}$ de la Kansas River à Kansas City en 1903 (maximum prévu $4.000 \mathrm{~m}^{3}$ ). Un autre conférencier, M. Rao, a cité le cas d'une rivière indienne, la Cauvery, qui a débilé, en $1924,456.000$ pieds cubes/s, alors qu'on n'avait prévu que 250.000 pour un projet de barrage (la crue ayant modifié le site, le projet a dù être revisé).

\section{DÉFINITION D'UNE CRUE.}

Quelques auteurs ont souligné la complexité de la notion même de "crue »: mème si on se borne à considérer une seule slation sur un cours d'eau, une crue n'est pas seulement un chiffre de débit maximum, mais elle comporte aussi l'évolution de ce débit en f́onction du temps, au cours d'une période de durée variable. Cette remarque a une importance considérable pour l'étude des dégâts causés par un débit dépassant la capacité des déversoirs. Une tentative pour analyser la forme de l'onde de crue a ćté faite notamment par M. Brither (Tehócoslovaquie).

La pinpart des auteurs ont admis, explicitement ou non, qu'on peut se borner à caractêriser une crue par le débit maximum atteint. Même dans ce cas, la définition reste ambiguë. On peut, par exemple, dire qu'il y aura crue chaque fois que le débit dépassera un niveau fixé d'avance. La plupart du temps on considère la « crue annuelle » qui est le plus fort débit atteint dans l'année. Signalons encore une légère ambiguïté résultant de l'époque choisie pour faire débuter l'année (année civile ou année hydrologique).

Certains ont cherché à assigner aux crues une limite physique, c'est-à-dire une valeur du débit qui ne peut être dépassée. D'autres ont affirmé qu'un tel concept est dépourvu de sens. Ce qui est certain, c'est que le débit d'un cours d'eau n'est pas rigoureusement illimité : M. PARDÉ a souligné l'absurdité qu'il y aurait à admettre pour la Seine à Paris des débits de $50.000 \mathrm{~m} / \mathrm{s}$. (Heureusement il n'est aucune des lois de probabilité effectivement utilisées par les hydrologues qui n'attribuerait à ce chiffre de $50.000 \mathrm{~m}$ : une probabilité astronomiquement petite; nous reviendrons là-dessus.) On doil ajouter que tous ceux qui ont cherché à atteindre effectivement la limite physique des débits se sont heurtés ou bien à des incertitudes immenses, ou bien à des valeurs de crues trop fortes, done assez invraisemblables pour qu'on soit justifié à substituer à ces « crues maximum possibles » des « crues maximum probables $»$. Un certain nombre d'Américains notamment ont été conduits à cela : nous citerons particulièrement Rissbol et Ryoflr, qui, se référant au « Standard Project Flood » adopté généralement, selon M. Rydelx, par le Corps des Ingénicurs américains, définissent la «crue maximum probable » comme celle provoquée par la chute des précipitations les plus défavorables qui se puissent rencontrer, sur un bassin possćdant des conditions d'écoulement "moyennes » (et non pas les plus défavorables).

\section{Aspect ÉCONOMIQUe DU PRORLAME.}

Si l'on veut envisager une solution plus précise, il faut résoudre dans chaque cas particulier un problème économique très complexe, consistant à mettre en balance des dépenses certaines, nécessitées par les ouvrages de protection, el les dégâts éventuels, difficiles à évaluer, que pourrait provoquer par hasard l'apparition d'une crue catastrophique. Ce point tres important a été souligné par un nombre notable d'auteurs. MM. Dinel et Bhanchet l'ont schématisé de la façon la plus claire, en proposant le secours d'une méthode graphique certainement apparentéc aux travaux de M. DANer sur \& la répartition optimum des charges entre les groupes d'une centrale hydro-électrique ».

C'est M. Riesbol qui a donné à l'examen du problème économique la place la plus large. Ii a fait une description très détaillée des dégâts qu'il faut envisager : pertes matérielles de divers ordres, pertes en vies humaines, et même perte de prestige pour le maitre d'œuvre qui fixe les risques à courir. Si l'on tient compte correctement de tous ces éléments, on peut con- 
sidérer le coût d'un déversoir comme une assurance. La notion de la rentabilité de cette assurance est primordiale. Il est entendu que pour obtenir une absolue sécurité, le coût des ouvrages serait en général prohibitif (1), M. RıssBOL reconnaît qu'en pratique l'emploi d'une méthode actuarielle intégrale pour fixer les risques à courir n'a encore jamais été tenté, l'ampleur des calculs étant excessive. Aussi, après avoir souligné en détail toutes les considérations qui, en droit, devraient intervenir, M. Riesisor conclut que le Bureau of Reclamation (U.S. Department of Interior) prend des décisions de façon assez synthétique, dépendant de la situation des ouvrages (régions très habitées ou désertiques par exemple) : on situera ainsi la crue à prévoir entre la crue maximum possible et la crue maximum probable. Deux autres auteurs américains, MM. Hathaway et Ryole, ont développé des considérations recoupant assez bien celles que nous venons de résumer.

Dans un opuscule intéressant à bien des égards, l'hydrologue néo-zélandais SchnackenBERG (2) signale les critères suggérés dans son pays il y a quelques années par une Commission de spécialistes, et nous en extrayons les recommandations suivantes :

Pour les projets de déversoirs de barrages, le Comité recommande les fréquences minimum suivantes :

1. Barrages font la rupture entrainerait des risques serieux de perles de vies humaines:

$a$ :

Barrages en terre ef en enrochements et tous barrages soumis at un risque de destruction rapide dépassant la valeur prévue.......... 1.000 ans b:

Barrages en béton et en maconneric, non soumis à un risque de destruction rapide $\ldots \ldots \ldots \ldots \ldots \ldots$

500 ans

2. Barrages ayant un coût de remplacement élcvé, dont la rupture n'entrainerait pas de risque sérienx de pertes de vies humaines........

3. Barrages ayan un coût de remplacement modére, dont la rupture n'entrainerait pas de risque séricux de pertes do vies humaines.......

!. Barrages ayani un fable coût de remplacement dont la rupture n'entrainerait pas de risque sérieux de pertes de vies humaines.........

500 ans

100 ans

20 ans

(1) « L'exces de prudence dans le choix de la crue "administrative», constate M. Giguet, appaurit la collectivité aussi sû̀ement que le femit nne faute technique... » (Travanx, mai 1951.)

(2) Schnackenberic. - « Extreme flood discharges 》, Yew Zealand Institution of Engineers, Wellington, 1949.
Il peut être intéressant de rapprocher les normes que nous venons de citer, de l'attitude prise fréquemment dans un probleme tout différent : celui qui consiste à fixer la garantie admissible dans la fourniture d'énergie électrique; on dira souvent qu'on juge admissible, par exemple, trois défaillances par siècle.

Dans les deux cas, on a affaire en droit à un problème économique, du type "assurance 》: mème si les grandeurs entrant en jeu ne peuvent pas toujours s'exprimer en termes monétaires, il s'agit bien de mettre en balance des risques fort aléatoires, avec les frais certains qu'entraîne le dispositif propre à nous prémunir contre ces risques. Et e'est bien à cause de la diversité des risques que les normes citées plus haut constituent une gamme assez ouverte.

Dans le mème ordre d'idées, nous signalerons que M. Coutagne, reprenant une idée développée par M. Borel (et d'ailleurs déjà exprimée par BUfFon), propose d'opérer de facon très synthétique en admettant de courir des risques comparables à ceux que l'on accepte en général dans la vie courante $\left(10^{-6}\right.$ d'après Boncr). Pourtant, MM. Drovhin, Marcet et Pacouant font observer que le public admet plus facilement la possibilité d'accidents en matière de voiture, de chemin de fer ou mème d'avion qu'en matière de grands barrages. Le public a pent-être quelque espèce de raison si l'on songe que bien souvent les dégâts sont à des échelles toutes différentes.

\section{QUALTE DES DONNEES D'OBSERVATION.}

Avant d'en finir avec cette introduction un peu longue peut-être, nous voulons signaler que plusieurs auteurs (comme MM. Hunter el Vrinot, PARDÉ, etc..), ont insisté avec raison sur l'attention qu'il faut accorder avant toutes choses a la qualité des relevés sur lesquels seront fondées les évaluations - qu'il s'agisse de relevés de débits ou de précipitations. On connaît trop les difficultés auxquelles se heurte cette exigence. C'est ce qui justifie pleinement la mise en garde de M. Coutagne selon laquelle « le probleme no comporte pas de solution générale à priori, ct doit par conséquent être étudié et résolu dans chaque cas particulier, compte tenu de toutes les données expérimentales de toute nature... que l'on possède sur le cours d'eau et son bassin, et en s'inspirant plus ou moins, pour suppléer au manque ou à l'insuffisance des données d'observations locales, de certaines considérations générales, de certaines formules statistiques et empiriques résultant d'observations et constatations faites sur d'autres cours d'eau de la région et mème d'autres régions $\gg$. M. PArdé, quant 
à lui, a longuement insisté aussi sur lintérèt qu'il y aura fréquemment à faire des recherehes historiques approfondies pour multiplier les informations, pourvu que ces recherches soient faites avec un sens critique averti.

Les nombreuses méthodes d'attaque exposées se classent assez bien en trois grandes catégories que nous désignerons, faute de termes meilleurs, par formules empiriques, méthodes statistiques et méthodes analytiques. On verra qu'en réalité ces méthodes sont loin d'être en concurrence : chacune d'elles s'appuie sur un choix délibéré, limitant arbitrairement les informations prises en compte. Cette constatation fait apparaître la nécessité aiguë d'une synthèse. Certes, un travail énorme reste à accomplir, mais on peut déjà donner quelques idées générales propres à guider le chercheur dans cette direction.

\section{II. - L'EMPLOI DE FORMULES EMPIRIQUES}

Sans que ce soit là une règle absolue, on peut dire que les méthodes que nous classerons dans ce chapitre mettent surtout l'accent sur les informations d'ordre général ressortant de la comparaison d'un grand nombre de bassins.

\section{Emplot D'Un COEFficient de SÉcurité}

Il en est ainsi implicitement de la méthode la moins élaborée, qui consiste dans chaque cas particulier à appliquer un coefficient de sécurité à la plus grande crue connue. Les renseignements retenus sur la rivière considérée sont réduits à cette unique crue, et la véritable justification de la méthode consiste dans son application plus on moins homogène à un grand nombre de bassins. Cette méthode est très employée : les Australiens, selon MM. Bard et Me Ildwratth, l'emploient concurremment avec d'autres méthodes, en tenant compte dans le choix des coefficients de sécurité de l'effet des réservoirs. M. Jorsinovic nous apprend que les Yougoslaves choisissent des coefficients de sécurité dépendant de l'importance des ouvrages. M. DuFraut souligne également l'emploi de cette mélhode en France.

Je n'insisterai pas trop sur le danger qu'il y aurait à fixer une fois pour toutes le coefficient de sécurité à employer, sans tenir aucun compte des conditions particulières (observations portant sur 10 ans ou sur 150 ans, cours d'eau alimenté par un glacier ou par des averses méditerranéennes, etc.); mais il faut attirer l'attention sur le fait que la façon dont on le fera varier constitue un problème extrêmement délicat : ce choix doit intégrer en effet les éléments économiques auxquels nous avons fait allusion, ainsi que tous les renseignements qui peuvent nous éclairer sur la fréquence probable, non seulement de l'unique crue retenue, mais aussi des crues plus importantes. Aussi, on peut penser que, dans bien des cas, il y aura intérêt à pousser un peu plus loin l'analyse. C'est l'objet des méthodes que nous allons signaler maintenant.

\author{
FORMULES FAISANT INTERVENIR \\ LES CaraCtéristroues DU BaSSIN
}

Elles sont nombreuses, et M. DAvis (Handbook of Applied Hydraulies) n'a pas en de peine à en réunir près d'une cinquantaine, toutes différentes, cette énumération étant sans doute Ioin d'ètre complète.

Ces formules traduisent en principe les enseignements tirés de l'étude d'un grand nombre de bassins, ou encore déduits par voie analytique des caractères hydrauliques de l'écoulement) mais ne sont en général valables que pour des régions très limitées, lorsqu'elles sont entièrement spécifiées numériquement. Elles visent à exprimer, soit une crue considérée comme maximum, soit plus généralement une crue assez respectable, ou encore correspondant en principe à une probabilité fixée.

Elles font intervenir successivement, lorsqu'on va des plus simples aux plus complexes :

- L'aire du bassin, généralement affectéc d'un exposant inférieur à 1 , souvent ćgal à 0,5 : formule de Mren, Kurchling, Gangitrlet, elc. - parfois à 0,75 : Diciens (Inde), WoOd (N.Z.), etc.

On trouve aussi : $2 / 3$ (Cooley) - 0,75 (Mretcalf, Eody), - 5/6 (Fanning).

-- Sa forme, en général, grâce à l’introduction d'une longueur, temps de parcours, ou temps de concentration : formules de Craig, Dredge, Burge, ete.

- M'intensité de la pluie : Hering, Petris, ete.

-. La pente du bassin : Burkli, Ziegler, CraMER.

- Enfin un certain nombre de formules introduisent la fréquence des crues exprimées : Frller, Horton, Lane, Creager, ete.

Dans cette dernière catégorie, certaines formules (Fulder, Maldet) condensent un raisonnement d'ordre statistique, leur forme analyti- 
que étant déduite de certains types de lois de probabilité.

L'emploi de toutes ces formules sera en général dangereux chaque fois qu'on ne connatra pas de facon approfondie les conditions dans lesquelles elles ont été établies.

Les Américains se sont livrés à une étude très poussée de la formule la plus simple, celle de Myer :

$$
\mathrm{Q}=\mathrm{CA}^{\alpha}
$$

$Q=$ crue à craindre.

$\mathrm{A}=$ aire du bassin.

\% étant en général pris égal à $1 / 2$, on a tracé pour les Etats-Unis des cartes du coefficient C, dit « cote Myer». Ces cartes montrent des variations tris capricieuses (1). Un travail analogue a été eftectué dans un certain nombre d'autres pays.

La communicalion de M. Tonns cite un nombre considérable de formules de ce type, établies par les hydrologues italiens. Le choix des coefficients a donné lieu à des études assez poussées pour les bassins italiens, et M. ToNini souligne les danger's que rencontrerait une extrapolation inconsidérée à d'autres régions.

M. Coutagne a cité de son côté, avec les mêmes réserves, quelques formules assez complexes. Il insiste sur leur caractere tres approximatif.

La communication de M. RaO a le mérite d'indiquer, pour une trentaine de formules de ce genre, un tableatu comparalif des résultats obtenus sur un fleuve indien. Ce tableau montre la dispersion énorme des résultats.

Les critiques de M. Parót à l'égard de l'emploi systématique de formules empiriques vont dans le même sens : il signale par exemple que les records constatés pour la Loire seraient de? à $10.000 \mathrm{~m}^{3}$ au Bec d'Allier, contre seulement 6.000 à 6.500 à Nantes. Il insiste en délail sur un certain nombre de difficultés de ce genre.

Signalons enfin que des formules empiriques du même type ont été étudiées dans le domaine des averses maxima : entre autres, l'exposé de MM. Bard el Me Ilowrarth signale les formules établies empiriquement pour l'Australie, faisant intervenir en mème temps que la fréquence la durée des arerses.

\section{LA MÉTHODE DES COURIBS RNYELOPPES}

Si nous avons réservé une place à part à la méthode dite des « courbes enveloppes», ce n'est

(1) Voir par exemple : Mernzen, MlJdrolog! (N.Y., 1949), p. 458-459. Un des principaux inconvénients de ce genre d'emploi systematique, c'est qu'on n'a pas trouvé jusqu'ici le moyen de tenir compte simplement de la diversité - en général très grande - des périodes de relevés. pas qu'elle soit plus précise, bien au contraire. Mais elle apporte des renseignements extrênement généraux pour une région donnée et elle a donné lieu à quelques remarques intéressantes.

Tous les traités d'hydrologie américains récents contiennent cet abaque assez familier, dans lequel on a porté, avee des échelles généralement logarithmiques, les plus fortes crues spécifiques observées en fonction de l'aire des bassins.

Les points ainsi représentés ne semblent pas dépasser une position limite, à laçuelle divers auteurs ont essayé d'ajuster des courbes analytiques (Mrer, Creagrer, ete.) (1).

Un travail analogue a été fait pour divers pays (Mexique, Suisse, Australie, Nouvelle Zélande, etc.) et la position des courbes limites s'est avérée varier considérablement.

Cela a permis à MM. Banil el Me Ildwrartu de proposer ingénieusement, pour les rivières australiennes sur lesquelles ils n'ont aucune information, de choisir pour valeur de la crue type un point situé entre les courbes enveloppes relatives à l'Australie et aux U.S.A., la position de ce point dépendant de l'importance des dégâts encourus. Ajoutons que les Australiens, en gens avisés et prudents, ne paraissent utiliser cette méthode assez originale que concurremment avec d'autres procédés d'estimation.

M. Couthave donne dans sa communication des abaques de ce type pour diverses régions de France, en y portant tantòt la crue maximum observée, tantòt le débit maximum des ouvrages d'évacuation. Les résultats, bien que fort intéressants, sont moins frappants que ceux des Américains, qui ont pu réunir des quantités beaucoup plus grandes d'observations.

Enfin, M. Riesbol, ayant signalé ce genre d'abaques, nous met judicieusement en garde contre les interprétations qu'on pourrait donner à la « courbe enveloppe» : il rappelle qu'au fur et à mesure que les années passent, des crues catastrophiques encore jamais observées arrivent à se produire parfois, qui obligent à déplacer la courbe limite. Et il ajoute « qu'aucune indication qu'une limite supérieure ait été atteinte n'est encore apparente $\gg$.

Nous venons d'exposer des méthodes qui semblent assez couramment employées, et qui mettent plus ou moins fortement l'accent sur l'emploi d'informations provenant de l'étude d'un certain nombre de bassins -. ce que nous pourrions appeler des renseignements d'ordre global.

D'autres méthodes, laissant au second plan cette catégorie d'informations, s'attachent à uti-

(1) Cit. SChNaCkenteri, « Extreme flood discharges », Wellington, 1949. 
liser au maximum les seuls relevés de débits de la station pour laquelle on cherche à évaluer la crue : on est ainsi conduit à un large emploi des techniques statistiques, et nous allons faire une revue des diverses variantes de cette méthode.

\section{III. - LES MẼTHODES STATISTIQUES}

L'idée la plus naturelle consiste à prendre les crues annuelles observées sur les $\mathrm{N}$ années dont on dispose, et à y ajuster une loi de probabilité.

A cette méthode est resté souvent attaché le nom de HAZEN, cité à New-Delhi par plusieurs auteurs. Sa mise en œuvre peut s'effectuer soit par un procédé purement graphique, soit par l'intermédiaire d'un type analytique de loi de probabilité.

\section{LE PROCÉDÉ GRAPHIQUE}

Le procédé graphique comporte lui-mème diverses variantes, suivant le choix des échelles : valeurs naturelles ou logarithmes pour les débits, et pour les probabilités, valeurs naturelles, logarithmes, temps de récurrence ou «probit», e'est-à-dire variable normale réduite correspondant à chaque probabilité. L'extrapolation se fait généralement par une droite, ajustée soit sur l'ensemble des observations, soit sur la partie supérieure. Il est à peu près évident que les résultats dépendent beaucoup de la méthode utilisée. M. Lx Cam avait essayé l'an dernier de donner une idée des erreurs d'échantillonnage. Le calcul en est évidemment complexe, mais des expériences ont permis d'y suppléer, et on a pu montrer que si l'on part d'une loi de Galton, avec 25 observations, l'écart-type de l'estimation graphique de la crue millénaire est sans doute supérieur à $100 \%$ de la valeur estimée.

\section{Les loIS LES PLUS EMPLOYÉES}

On réduit l'arbitraire par le procédé consistant à ajuster une forme analytique de loi de probabilité. Les types effectivement employés sont nombreux, nous énumérerons les principaux.

La loi de Gauss est parfois employée, mais généralement on la rejette à cause de sa symétrie : c'est une constatation empirique très générale que la distribution des crues est dissymétrique.

La loi de Galton, citée ou employée notamment par MM. Hunter et Wilnot, Covtagne, Mallet, Gibrat, Delattre et Henry, Parisot et Roprer, etc., traduit bien cette dissymétrie. Mais il semble que son comportement asymptotique traduise une décroissance trop lente dans certains cas. Aussi a-t-on souvent utilisé la loi de Gumber, plus satisfaisante à cet égard (Coutagne, Gibrat, Bose et Nag, Parisot et Rodier). Nous reviendrons sur les justifications théoriques qui ont été données à ces deux derniers types de lois. Sans prétendre être exhaustif, on peut compléter cette énumération par quelques autres lois effectivement utilisées : Première loi de Laplace. - Types de Pearson (et particulièrement le type III utilisé par l'éminent hydrologue américain Foster). - Lois de SladF, dérivant de la loi de Galton par des substitutions homographiques. - Lois de Goopricri, dérivant de la loi de LAPLACE par des transformations simples. - Enfin lois de M. Halphes dont nous avons parlé il y a deux ans à la S.H.F. (1).

Il est intéressant de comparer les différences entre les comportements asymptotiques de toutes ces lois. Ces différences sont plus apparentes si on exprime les crues en fonction de leur période de récurrence, comme l'a fait notamment M. CouTAGne. Nous empruntons les résultats à une étude de M. LE CAM :

Loi de Gauss..... Q $\sim \sqrt{\log T}$

Loi de Laplace. . . Q $\sim \log \mathrm{T}$

hoi de Galton.... Q $\sim \exp \sqrt{\log T}$

Loi III Pearson. . $\quad Q \sim \log \mathrm{T}$

Lois de Salde.... Q $\sim \frac{a \exp .}{c \exp }\left(\frac{\sqrt{\log \mathrm{T}}}{\sqrt{\log \cdot \mathrm{T}}}\right) \frac{+b}{+d}$

Loi de Gumbol. . . Q $Q \sim \log \mathrm{T}$

Loi de Gooprrch... $Q \sim\left(\frac{\alpha \log T+\beta}{\gamma \log T+\delta}\right)^{i / c}$

Lois de HaLphen.. $\begin{cases}Q \sim \log T & \text { pour le type } A \\ Q \sim \sqrt{\log T} & \text { pour le type } B\end{cases}$

Bien entendu nous n'exprimons là que l'allure du comportement asymptotique. Dans un cas par-

(1) « Les lois des débits des rivières françaises 》 (LE Can et Morilat'. 
ticulier, les écarts obtenus par l'utilisation de deux de ces lois dépendront des procédés d'ajustement respectivement utilisés. Un graphique établi par M. Le CaM (fig. 1) permet de comparer

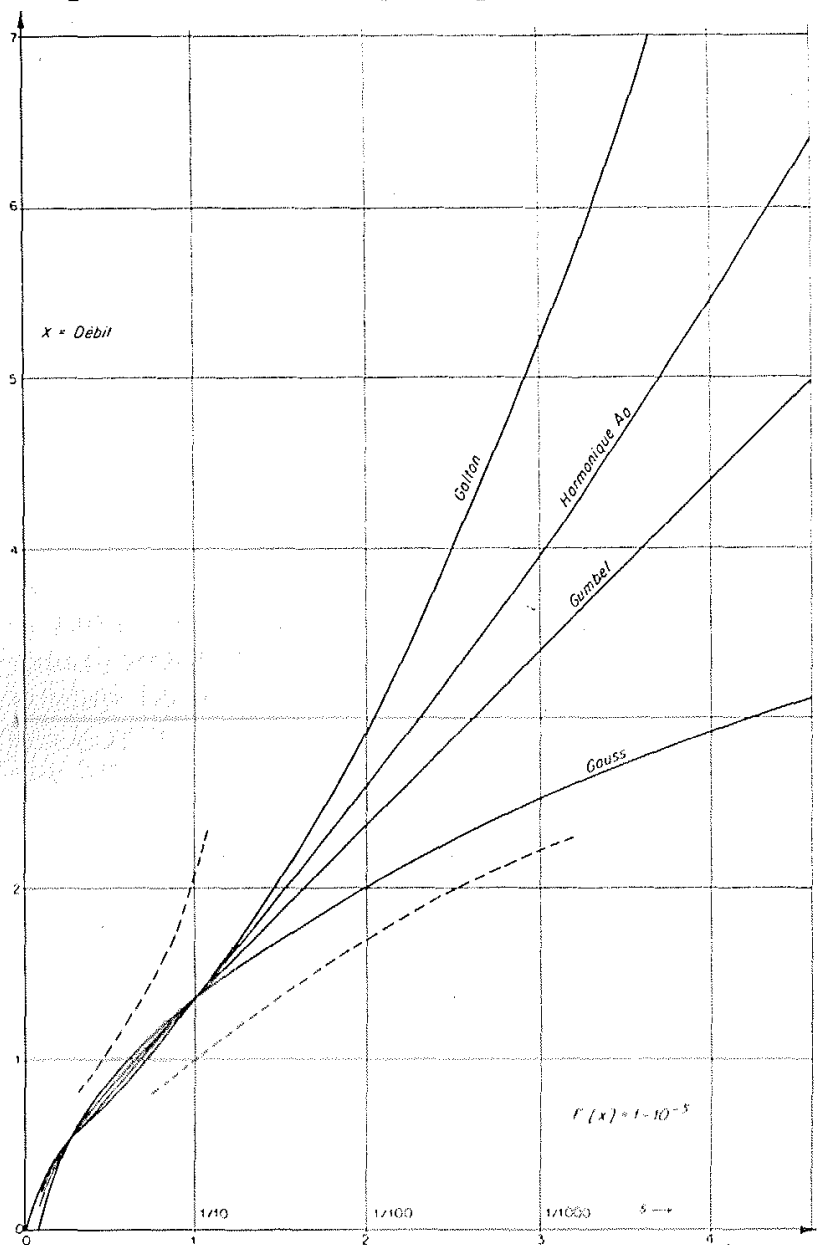

Fri. 1. - Ce graphique est destiné à montrer la difference du comportement asymptotique de diverses lois. On a fait coincider les points de probabilité $1 / 2$ at $1 / 10$. Les courbes en pointille sont les courbes de confiance a $95 \%$ sur la variable s lorsque l'on a un échantillon de 40 points représenté expérimentalement par une loi voisine de celle de Gumbl.

quelques-unes de ces lois lorsque l'ajustement fait coincider les points de probabilités $50 \%$ et $10 \%$. Par ailleurs, la communication de M. CovTaGNe contient des graphiques analogues relatifs à deux rivières américaines : Columbia River et Kansas River - les procédés d'ajustement n’étant peut-être pas précisés de facon complète.

\section{ReMARQUES GÉNERALES SUR IES MÉTHODES STATISTOUES}

L'emplọi de ces lois de probabilité a été parfois critiqué, en particulier par M. PardÉ, qui a donné à New-Delhi un certain nombre de remarques fort judicieuses. Mais nous croyons bon de souligner que la plupart des critiques valables qui ont été avancées à l'encontre des méthodes statistiques résultent de malentendus. A la question que pose M. PARDÉ : « Tout d'abord, est-on sûr que les possibilités de tous les cours d'eau, en ce qui concerne les grandes inondations, sont intégrées et inscrites, incorporées comme sont semés des germes, dans les parties basses, expérimentales des courbes? $\gg$, à cette question qui touche au cour même du problème, on doit répondre par les observations suivantes : lorsqu'on dispose de sources d'informations valables autres que les relevés de crues, alor's certainement les méthodes dont nous venons de parler, n'utilisant pas ces informations, ne constituent pas ce qu'on peut faire de mieux. Mais si, au contraire, nous ne savons rien d'autre que les valeurs des crues observées pendant $n$ années ou si nous avons décidé de n'utiliser rien d'autre, alors l'extrapolation d'une courbe de probabilité judicieusement ajustée parait être la seule chose possible, et d'ailleurs M. PARdé l'admet comme nous, semblet-il. Que cette extrapolation soit hasardeuse, soit. Mais aucun statisticien ni personne d'autre à notre connaissance, ne saurait faire sortir un lapin d'un chapeau qui ne contient que de la farine. Dans le cas simple où on peut répondre par « oui » à la question de M. PArdê, j'ajouterai que les statisticiens cherchent à chifrrer ce carac-

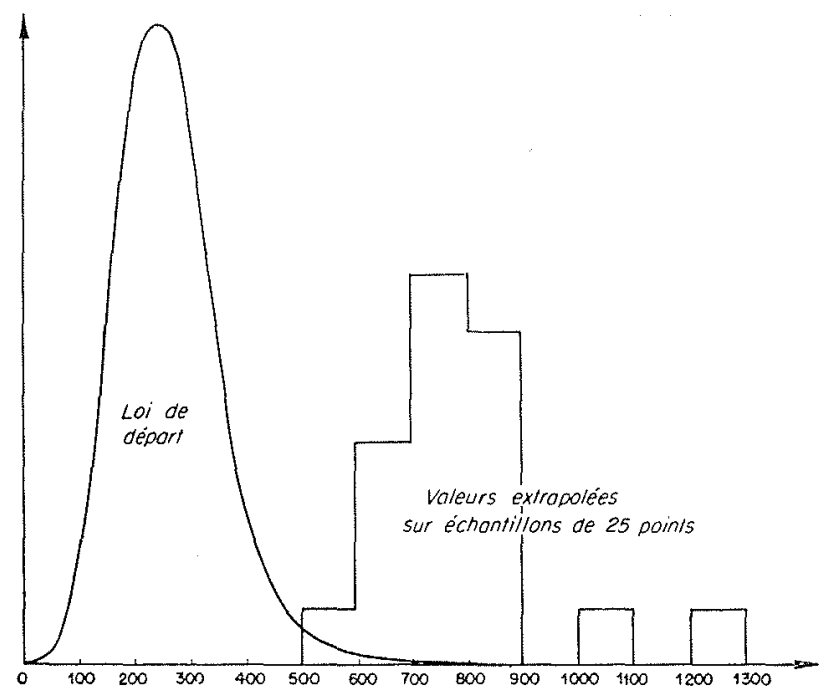

Fig. 2. - EnReurs D'ichanthlionnage dans r'Évaluation

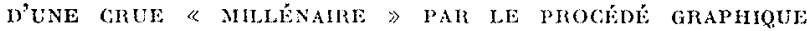
HABTUEL.

(Répartition expérimentale de 20 valeurs extrapolées a partir d'echantillons de 25 points.)

La loi de départ est ici un type III de Peanson. On a construit des échantillons de 25 points à l'aide des tables de Frsher et Yarss (Random numbers). L'extrapolation graphique pour la crue millénaire effectuée sür 20 séries distinctes, a donné les valeurs dont Ia distribution est figuree par le polygone de droite. On constate une dispersion assez notable. 
tère hasardeux, en calculant ce qu'on appelle les erreurs d'échantillonnage. Un tel calcul s'avère extrêmement complexe, en général, dans le problème des crues, mais on peut en donner des solutions approximatives (voir fig. 2).

Rappelons aussi que les instructions donnces aux ingénieurs américains par le Geological Survey (qui paraissent constituer un document semiofficiel bien que ne devant pas être rendu public) font état d'une méfiance très nette à l'égard des méthodes statistiques. Peut-être le développement des méthodes analytiques, basé sur l'étude de l'écoulement des fortes averses, est-il assez poussé aux Etats-Unis pour que les autres méthodes prennent en apparence une importance secondaire. Mais ce n'est peut-être pas exact en général.

\section{Mise EN GARDE}

CONTRE QUELQUES DANGERS FRÉQUENTS

Avant de clore cette revue un peu rapide des méthodes statistiques courantes, il semble indispensable de rappeler un certain nombre de points délicats, qui ont conduit des auteurs insuffisamment prévenus à des affirmations diseutables, et parfois inexactes.

On peut d'abord remarquer d'une facon générale que certains hydrologues auraient intérêt à avoir une notion claire de ce que les statisticiens appellent "erreurs d'échantillonnage » dont nous venons de parler. C'est un point sur lequel M. PARDÉ a Ionguement insisté, quoique en un langage tout différent. Cela éviterait des affirmations faites sans précaution, comme celle de ces auteurs qui conseillent, dans les ajustements statistiques des séries de crues, d'éliminer systématiquement les points qui paraissent aberrants. Le danger d'une telle consigne n'a pas besoin d'ètre souligné.

Ensuite, nous indiquerons l'utilité qu'il y a à ne pas oublier que les débits des différents jours de l'année sont pour la plupart des rivières, très loin de suivre la mème loi de probabilité, et aussi très loin d'être indépendants. Cet oubli rend parfois dangereux l'emploi de la méthode préconisée par M. Gibrat, qui consiste à prendre en compte tous les débits et non pas seulement les crues annuelles. Si ce procédé pouvait améliorer l'information dans le cas où M. GrBRA'T lui-même l'a utilisé (en particulier pour la 'Truyère), par contre on peut facilement imaginer que son emploi inconsidéré pourrait induire en erreur : il suffit de penser à un cours d'eau présentant des hautes eaux d'été très prédominantes, mais très régulières parce que d'origine glaciaire - et ayant systématiquement ses crues en automne et en hiver, par suite de la présence d'un bassin pluvial à régime beaucoup plus irrégulier. Alors le mélange de tous les débits risque de fausser systématiquement l'extrapolation (roir fig. 3).

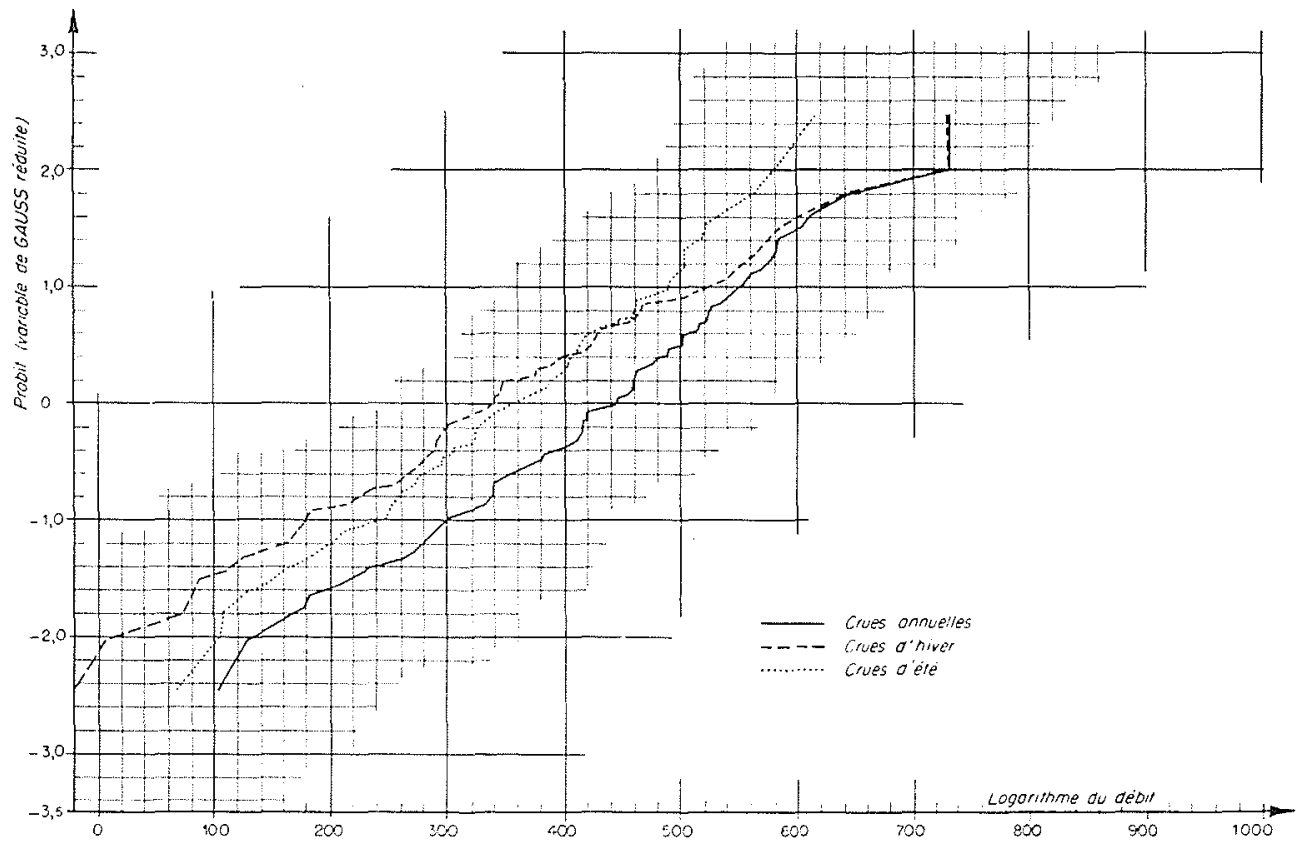

Fici. 3. - Crues du Rhin a Strasioura. 
D'autre part, il ne faut pas non plus oublier l'existence de corrélations spatiales (entre les crues de divers bassins) dont le rôle est parfois essentiel : ainsi des auteurs ont affimé qu'ils pouvaient suppléer à la brièveté des relevés historiques pour tel bassin, en y joignant les relevés d'un certain nombre de bassins voisins. II est bien clair que si l'on a afraire à une région très homogène, tel le Massif Central, c'est là une dangereuse illusion de sécurité.

Terminons en soulignant les malentendus qui sont à l'origine des privilèges souvent attribues a certains types de lois, dites «lois théoriques» ou « lois ayant un fondement rationnel».

M. Gibrat a lui-même rappelé, dans sa communication à New-Delhi, que la loi de Galton dont il a préconisé l'emploi constitue seulement « une représentation mathématique convenable de la courbe des débits classés ». Mais beaucoup des auteurs qui ont utilisć cette loi après lui ont cru y voir la « loi véritable » des débits, prenant a la lette ce qui n'elail chez M. Gmant qu'une simple suggestion, à savoir le fait que cette loi pouvait résulter du processus qu'il a appelé l' « effet proportionnel ». Comme l'ont souligné MM. Pariso't et Rodier, il est encore loin d'être prouvé qu'un tel processus existe dans le cas des débits,

Bien entendu, cela n'enlève rien à l'intérêt de la loi de Garton, qui s'avère empiriquement représenter parfois correctement la répartition des débits.

Des illusions plus dangereuses semblent elre entretenues à propos de la loi do Gumbet. Rap- pelons que MM. Fisher el Trppett, qui ont les premiers introduit cette loi en 1928 , avaient souligné que c'était une position limite de la loi de la plus grande de $\mathrm{N}$ variables indépendantes, et suivant la même loi de probabilité, pour $\mathrm{N}$ très grand. Or, les 365 débits des jours d'une année sont très loin d'être indépendants, très loin de suivre la même loi, et aussi très loin d'être assez nombreux, puisque Fisher et TippetT (1), étudiant la rapidité avec laquelle cette loi limite est atteinte, ont conclu à une convergence extraordinairement lente : il faudrait un nombre de l'ordre du million pour avoir une bonne approximation (KENball, plus exigeant, cite le chiffre de $\left.10^{22}\right)$. On voit que nous sommes loin de compte, et les véritables justifications à l'emploi de la loi de Gumber ne samaient ètre qu'empiriques : ajoutons que cet emploi s'avère souvent satisfaisant.

Dans plusieurs publications, M. Gunber a donné les résultats obtenus pour quelques cours d'eau, en particulier le Rhône à Lyon ct le Mississipi à Vicksburg. On constate que l'ajuste. ment de la loi utilisée avec les observations est très bon dans le cas du Mississipi, mais assez discutable pour le Rhône (2).

(1) Limiting Forms of the Frequency Distribution of the largest or smallest Nember of a sample. - Proc. of the Cambridge Phil. Soc, vol. 24, p. $180,1928$.

(2) Voir, par exemple :

Gumber. -- "The return perriod of flood tlows》. Annals of Mathematical Statistics, 1941.

- "Theorie statistique des debits de moe z. Renatssames, N.Y., 1945.

\section{IV. - LES METHODES ANALYTIQUES}

Nous désignons ainsi les méthodes d'investigation dont l'objet est le mécanisme des crues. II s'agit de calculer au mieux la valeur du débit maximum auquel donnera naissance une averse donnée. Bien entendu, l'étude de ce mécanisme ne domne aucun renseignement par elle-même sur la probabilite des crues obtenues. Les averses dont on part sont le plus souvent considérées comme les averses maximum pouvant se produire sur le bassin, mas on peut craindre qu'il n'y ait dans celle affirmation une incertitude considérable.

\section{LA PROBABILITÉ DEs averses}

L'étude de la probabilité des averses prises en compte est donc souvent négligée. Un certain nombre d'auteurs s'en sont pourtant souciés.
MM. Honter et Wrimot en onl fait une analyse assez qualiative, soulignant surtout les différences qui apparaissent dans les diverses parties du monde, leurs exemples portant sur la GrandeBretagne d'une part, la côte occidentale de l'Inde d'autre part.

Rappelons aussi les formules empiriques données par MM. Barro et Me ILLwrate pour l'intensite des averses maximum, en Australie, en fonction de leur durée et de leur temps de récurrence. Les mêmes auteurs ont constaté que la loi de Gatton s'applique bien à la répartition des plus fortes averses observées au cours de chaque année.

De leur côté, MM. Bose et Nag ont employè de façon satisfaisante la première loi de LAPLACE à la distribution "tronquée » des plus fortes averses.

Signalons enfin que M. Hathaway, qui met 
l'accent sur l'étude des averses dans l'évaluation des crues conformément à la tendance d'une grande partie des ingénieurs américains, indique, d'après les observations américaines, des valeurs d'averses maxima en fonction de leur durée (cf. fig. 4).

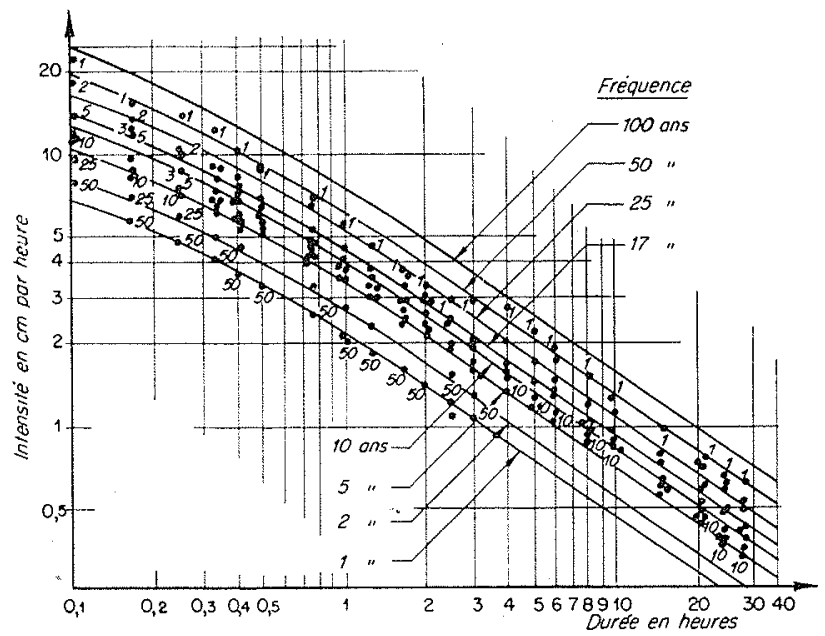

Fig. 4. - ETUde de la relation Fréguence-durée-intensité povr les plutes a Boston (Mass.), d'après Sherman.

Il serait bien agréable que nous puissions diposer un jour, en France, de graphiques analogues à celui-ci, établis pour un grand nombre de stations. Les Archives de la Météorologie Nationale contiennent sans doute les chiffres nécessaires à certaines études de ce type et il est à souhaiter que leur exécution puisse ètre abordée avec les moyens matériels nécessaires.

Remarquons que le graphique ci-dessus ne concerne que des précipitations ponctuelles. La répartition des averses dans l'espace pose un probleme plus complexe et non moins important (abordé d'ailleurs dans certaines études américaines récentes).

MÉThODEs DE «Transposition DEs AVERSEs 》

Lorsqu'on veut appliquer des méthodes analytiques à des bassins où l'on n'a pas observé en détail d'averses pouvant ètre considérées comme plus ou moins « maxima », alors on est conduit à $\mathrm{y}$ transposer des averses observées sur des bassins voisins. Les précautions à prendre dans ce genre d'opération ont été assez bien indiquées; M. Hathaway en expose diverses variantes.

Dans cet ordre d'idées, M. Prccon r signale que, d'après tous les renseignements qui ont pu être réunis sur les averses en Italie, si l'extension des données d'une station judicieusement choisie à un bassin d'étendue assez limitée s'avère valable sous certaines précautions, par contre, l'extrapolation des valeurs des averses d'un bassin à un autre, même voisin, lui semble très délicate : il a rencontré pour des bassins très voisins des différences de régimes fort sensibles. Peut-être cette constatation est-elle à rattacher au caractère, que nous dirons méditerranéen, du climat de l'Italie; on sait que ce climat est, d'une façon générale, beaucoup moins uniforme que les climats océaniciues.

Ajoutons enfin que M. PARDÉ, qui expose de facon brève mais claire le principe de ces transpositions, indique judicieusement que les bassins à comparer devront être choisis par « un climatologue averti ».

\section{RôLE DES INFLUENCES NIVALES}

Dans de nombreux cas, comme on l'a souvent observé, les crues catastrophiques sont causées par la coïncidence de fortes pluies et de fonte d'un important stock nival. Bien que les deux phénomènes soient assez étroitement imbriqués -- la fonte étant accélérée par des chutes de pluies relativement chaudes (par un processus du reste assez complexe, où certains effets mécaniques (déchiquetage) ont sans doute plus d'importance qu'un effet calorifique direct) - il y a pourtant intérêt à évaluer séparément leurs contributions au débit.

M. Hathaway a exposé de facon fort concrète les principes du calcul de la contribution nivale : division en zones d'altitude, évaluation par zone du stock nival, des conditions météorologiques et de la fonte résultante en utilisant des « équations de fonte $»$ établies par son compatriote Phillip LIGHT.

M. PArdé signale que vraisemblablement une quantité d'eau donnée produira une contribution plus importante à la crue si elle provient de fonte nivale que si elle est due à des averses actuelles : la présence de neige en fusion a chance de diminuer à la fois l'infiltration et l'évaporation.

\section{L'HYIROGRAMME UNITAIRE}

Enfin, la partie essentielle des méthodes analytiques réside dans ce que les hydrologues américains Shernan et Bernard ont appelé « l'hydrogramme unitaire ». Il s'agit de la technique qui permet d'évaluer, à partir d'une averse ou d'une contribution nivale donnée, l'augmentation des débits au cours du temps. Cette technique n'a peut-être pas été absolument «inventée » par les hydrologues cités. et M. PARdé rappelle qu'il a préconisé depuis longtemps des méthodes qui s'y rattachent étroitement. Mais les Américains ont eu le mérite d'en donner une version extrênement claire parce qu'assez schématisée, et d'en faire des applications systématiques très poussees (1).

(1) Voir par exemple dans Meinzer (op. cit.), le chapitre redige par Shemman : « The Unit Hydrograph method $»$, pp. 514-525. 
Sous sa forme la plus simple, la méthode de l'hydrogramme unitaire fait appel aux postulats suivants :

$1^{\circ}$ Sur un bassin saturé, une averse d'une durée unité et d'une intensité unité donne naissance à un « hydrogramme » qui ne dépend que de la forme du bassin;

$2^{\circ}$ La même averse, avec une intensité $i$, donne un hydrogramme obtenu en multipliant par $i$ les ordonnées de l'hydrogramme unitaire;

$3^{\circ}$ Des averses successives donnent lieu à un débit obtenu par addition des ordonnées de leurs hydrogrammes respectifs.

En langage mathématique, cela revient à dire qu'on passe des pluies aux débits par un opérateur linéaire.

Des variantes plus ou moins perfectionnées sont très nombreuses - en particulier on peut introduire les débits d'infiltration profonde et infiltration «hypodermique »( voir fig. 5).

De nombreuses études ont été faites en vue de relier les paramètres caractérisant l'hydro-

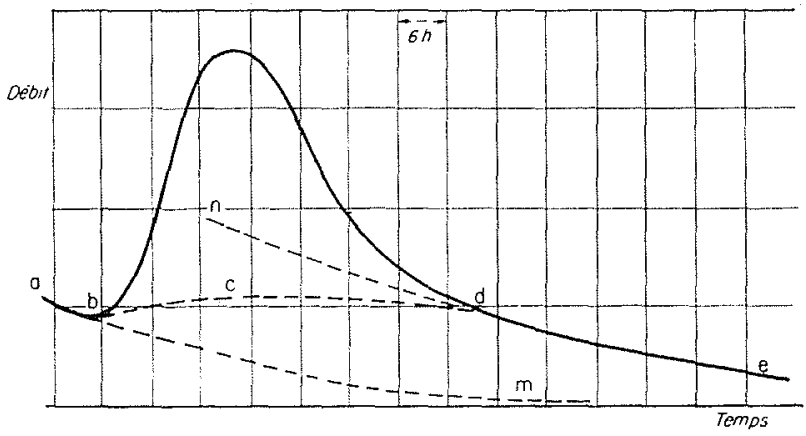

Fig. 5. - Construction SCHËATIQUE D'UN HYDROGRAMML UNITARE PAR LA METHODE DITE DE L'AVERSE ISOLÉE (d'après JOHNSTONE et Cross).

abm: débil de base (coulbe de tarissement précédant l'averse).

$n$ de: courbe de larissement après la fin de l'averse.

$b$ c $d$ : compte tenu de l'infiltration, la partic du débit située au-dessus de cette courbe représente l'ecoulement superficiel.

gramme unitaire aux caractéristiques du bassin (Bervard, Mc Carthy, Snydor, Clark, etc...). Nous ne pouvons les exposer ici en détail. Remarquons seulement ceci : en pratique, on détermine

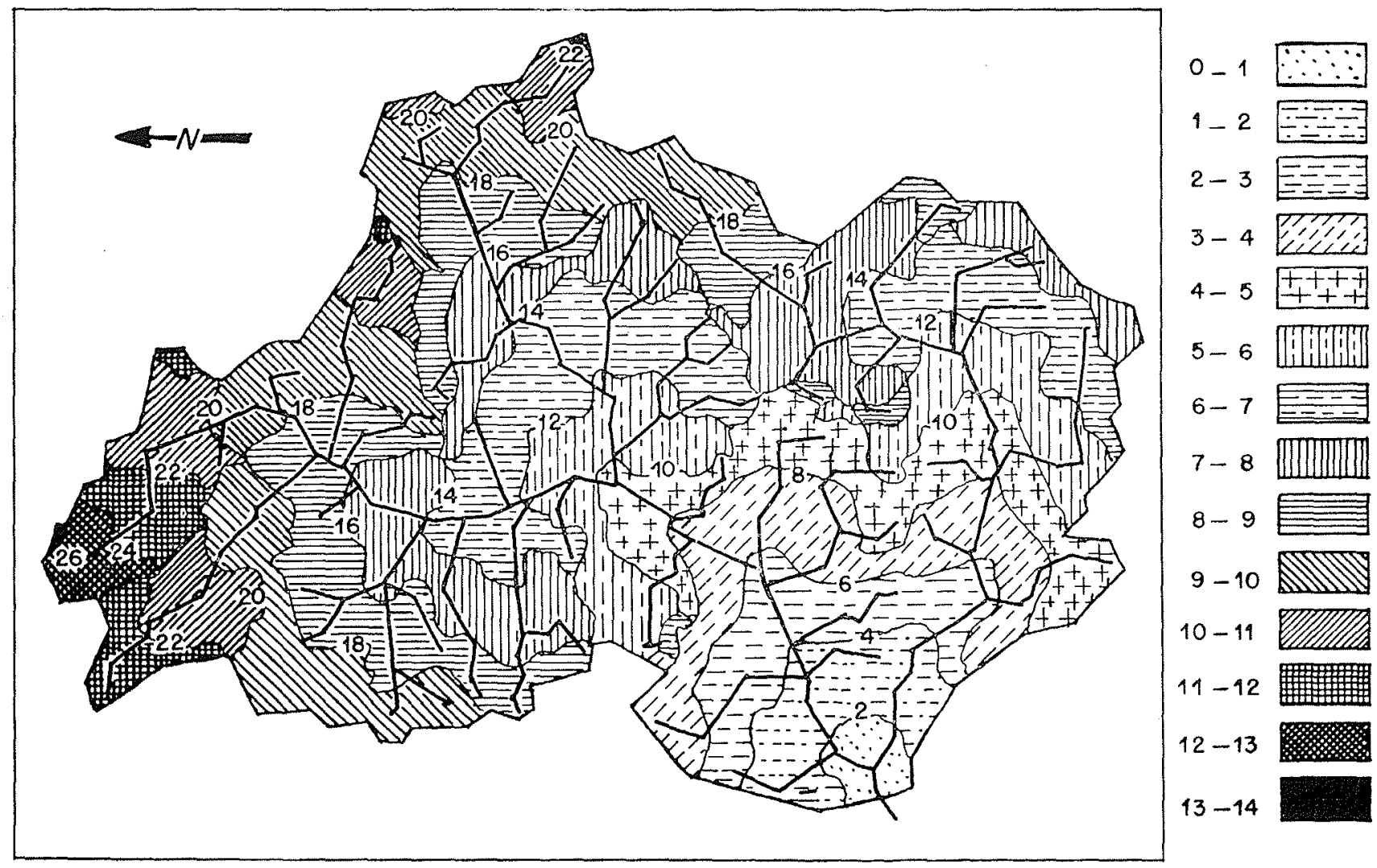

Fig. 6. - Bassin de ta Haute Duhance.

Courbes d'égal temps de parcours dans lhypothèse de vitesses de propagation uniformes.

Nous mettons actuellement au point un programme d'étude détaillée de crues de la Haute Durance. Ce travail nécessitera au préalable un renforcement considérable des réseaux actuels d'observations, tant pluviométriques qu'hydrométriques: nous avons affaire en effet à un bassin caractérisé par une très grande hétérogénéité climatique. 


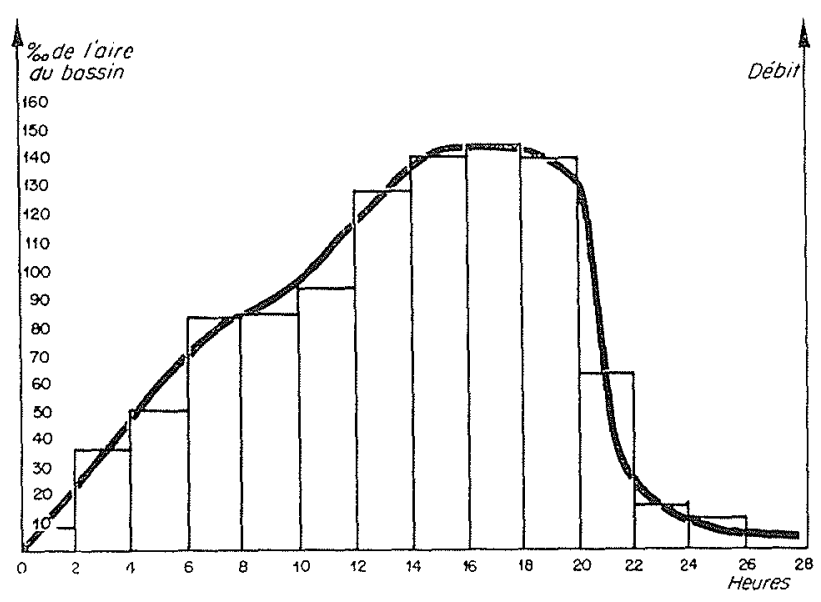

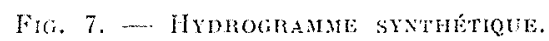
DURANCE à SERRE-PONGON.

Des courbes d'égal temps de parcoury de la figure 6 , on décluit d’hydrogramme ci-dessus, qui résulterait théoriguement d'une averse isolée très courte, uniforme su: tout le bassin: souvent de facon empirique l'hydrogramme unitaire en examinant le débit qui résulte d'une averse-type suffisamment isolée. La reconstitution des débits ultérieurs à l'aide de cet hydrogramme et la comparaison avec les observations fournit un controle automatique dont les conclusions s'averent souvent assez satisfaisantes. On peut aussi construire des "hydrogrammes synthétiques $\gg$ (cf. figs. 6 et 7 ).

L'exposé de ces méthodes on des applications variées ont été citées par un grand nombre d'auteurs. Nous nous bornerons à en énumérer quelques-uns : Bard et Mc Illwratph, HathaWay, Covtagne, Rao, Pardé, etc...

Les variantes les plus perfectionnées de ces méthodes conduisent à des évaluations assez précises, mais si on les emploie exclusivement on est souvent conduit, comme l'a observé M. LE Cam, à calculer avec une grande précision une crue dont on ignore la probabilité.

\section{V. - POSSIBILITẼ DE SYNTHESE PLUIES-DÉBITS}

Voici achevée une revue d'ensemble, très brève, des différentes techniques dont on a parlé dans les rapports présentés à New-Delhi. Sans ètre complet, nous pensons avoir eité les points les plus importants. La plupart des communications avaient évité des détails trop techniques. Nous avons accentué encore ce souci, ce qui confère à cet exposé un aspect parfois un peu imprécis. Mais citer par exemple toutes les lois de probabilité utilisées, ainsi que leurs propriétés essentielles, ou encore toutes les techniques numériques de calcul des hydrogrammes unitaires, nous aurait entrainés trop loin.

\section{LE BESOIN D'UNE SYNTHÉSE}

Nous avons surtout tâché de dégager les idées générales, et pour ainsi dire la philosophie des différentes mélhodes citćes.

Les liens entre ces différentes méthodes ne sont peut-être pas très apparents. C'est que jusqu'ici on les a toujours négligés. Mais l'ordre dans lequel nous avons reconstruit l'ensemble des communications de New-Delhi nous permettra tout à l'heure de montrer où peuvent s'insérer ces liens.

Auparavant, nous voulons signaler une impression qui se dégage à la lecture des divers rapports : il semble que les ingénieurs ou hydrologues qui ont cité une seule méthode d'approche, soit parce qu'ils n'en connaissent pas d'autres, soit parce qu'ils utilisent, par choix ou par nécessité, des ćléments d'information strictement délimités, il semble que ceux-là n'aient eu aucune inquiétude : la méthode qu'ils préconisent est la bonne. Mais il n'en est pas de même des hydrologues - heureusement les plus nombreux - qui ont cité ou employé simultanément diverses méthodes. Ceux-là semblent gènés pour décider si telle méthode vaut mieux que telle autre. Ils sont conduits pour la plupart à conclure comme les Australiens Bard et Me ILLwratrH qui assurent qu'en Australie on emploie simultanément 6 méthodes (formules empiriques - facteur de sécurité - courbe enveloppe -- lois de probabilité - carte pluviométrique - hydrogramme unitaire) et la décision tient compte des divers résultats.

Peut-ctre verra-ton qu'il y aurait quelque intérêt à soumettre à l'analyse le processus par lequel s'effectue cette combinaison. Mais il apparaît aussitèt que le problème doit être repris à la base (au moins théoriquement). En effet, les méthodes que nous avons groupées sous le titre un peu vague de «formules empiriques» utilisent exclusivement pour la détermination de la crue les informations à priori qui peuvent être suggérées par l'étude antérieure d'un certain nombre de bassins; les méthodes dites « statistiques $\gg$ se bornent au contraire aux statistiques de débits - et les procédés qualifiés d' « analytiques » tablent sur les données pluviométriques, en mettant l'aceent sur le mécanisme de passage pluies-débits, et en négligeant d'ailleurs assez souvent le côté « probabilité des averses ». 
Ces trois classes d'informations sont certainement les plus importantes, bien qu'on puisse parfois rencontrer d'autres renseignements valables. Dans la plupart des cas concrets, on se trouvera en présence d'informations de chaque sorte, et on imagine facilement que le traitement le plus efficace pour en extraire le plus d'information sur la crue qui nous intéresse ne soit peutêtre pas de traiter chaque catégorie de données comme si les autres n'existaient pas.

\section{LE SCHEMA PROBABILISTE « PLUIES-DÉRITS 》}

Pour combiner les diverses informations dont nous venons de parler, il est essentiel de disposer d'abord d'un schéma mathématique représentant la facon dont les pluies domnent naissance au débit. Des recherches effectuées par M. LE Cam ont ouvert là une voie très féconde. En gros, il s'agit simplement d'exprimer sous forme mathématique les hypothèses des méthodes d'hydrogramme unitaire : on dira que le débit dérive de la pluie par un opérateur linéaire :

$$
x(t)=\int_{-\infty}^{t} k(t-\tau) y(\tau) d \tau
$$

où $y$ représente la pluie et $x$ le débit.

M. LE Cam a pu vérifier, au moins dans quelques cas, que l'approximation obtenue est bonne. On peut d'ailleurs introduire des opérateurs plus généraux, mais les calculs sont plus complexes.

Il a joint à ce schéma de passage pluies-débits des hypothèses extrèmement satisfaisantes concernant la loi de répartition des averses. Ces hy- pothèses, dont les idćes essentielles sont dues at M. Halphen, constituent, avec un nombre de paramètres relativement faible, un schéma d'une grande souplesse, qualité qui s'avère ici indispensable (comme le montrent les recherches de M. Mineur, par exemple) (1). De ces deux schémas, représentant la distribution des pluies et le passage pluies-débits, il est possible de déduire la répartition des débits par des calculs purement mathématiques. Et nous signalerons un point qui mérite quelque intérêt : c'est qu'on obtient ainsi, pour la forme analytique des lois de probabilité des débits, certains types coincidant avec les lois proposées par M. HALPhEN, dont la forme avait été fixée au simple vu des débits. Sans tomber dans le travers consistant à dire que cela donne une justification théorique à nos lois (qui seraient alors « les vraies lois»), nous voyons là au moins une coïncidence heureuse.

On conçoit qu'en prenant pour base le schéma dont nous venons de donner une rapide ébauche, il soit possible d'aborder le problème de l'estimation d'une crue en combinant logiquement les renseignements valables qu'on peut posséder sur les pluies, sur les débits, et aussi sur les propriétés hydrauliques, topographiques, etc., du bassin, qui interviement dans la fixation des constantes de l'opérateur de passage pluie-débit. On peut faire par là la synthèse d'une classe assez large de méthodes.

(1) Mineon : Etude numérique de la chaîne des jours sees et humides au Parc-Saint-Maur : « Il apparaît... que la chaine... est au moins triple et sans doute d'ordre pratiquement infini. »

\section{VI. - LA NOTION DE PROBABILITÉ EN HYDROLOGIE}

\section{INFORMATIONS N'ENTRAN'T PAS DANS ILE SCHÉMA PRÉCÉDENT}

Pourtant, le schéma élargi dont nous venons de parler, qui constitue un progrès énorme par rapport aux solutions partielles envisagées jusqu'ici - et dont nous espérons que ses développements ultérieurs pourront donner des résultats fort remarquables - ce schéma ne semble tout de mème pas pouvoir intégrer toutes les informations valables qu'on peut avoir sur une crue. Nous allons examiner quelques catégories de renseignements qui n'entreraient qu'avec peine, ou pas du tout, dans ce schéma.

a) L'existence d'événements extraordinaires, jamais encore observés, mais réputés possibles.
On peut concevoir par exemple deux bassins de situations fort différentes, sur lesquels les crues moyennes et extrêmes observées seraient tout à fait comparables; mais il se peut que sur l'un d'eux seulement soit possible un certain phénomène, résultant d'un concours de circonstances atmosphériques, et donnant naissance à des débits cataclysmiques. Ce même phénomène pourrait être matériellement exclu sur l'autre bassin.

Nous avons appelé cela «problème du déluge ». C'est cette éventualité qu'exprime M. Pardé d'une façon assez frappante en disant : «Est-on sûr que les possibilités de tous les cours d'eau, en ce qui concerne les grandes inondations, sont intégrées et inscrites, incorporées 
comme sont semés des germes, dans les parties basses, expérimentales des courbes? »

Une façon plus mathématique d'exprimer la même idée consiste à dire que des distributions de fréquence ayant le même comportement semiasymptotique, n'auront pas ipso-facto le mème comportement asymptotique, (c'est-à-dire pour des valeurs plus éloignées de la région observée). Sous cette forme, on voit que le problème envisagé devrait avoir des répercussions sur le choix du type de loi de probabilité utilisé. Or, en pratique, on constate souvent que lorsqu'on possède des informations du genre de celles auxquelles nous faisons allusion ici, on en fait d'abord abstraction pour traiter le probleme statistique - quitte à dire : ces informations seront tout de meme prises en compte au moment de la décision, mais d'une facon extra-statistique. Nous verrons tout à l'heure que cette attitude est liée à l'interprétation philosophique de la notion de probabilité, et que ce n'est peut-être pas la plus souhaitable.

b) Une autre catégorie d'informations qu'on peut avoir parfois à envisager concerne l'évolution du climat. Il semble que pour les géographes comme M. Pardé, la possibilité d'une telle évolution ne fasse guère de doute . - Tout particulièrement, remarque M. PARDÉ, à l'échelle des longues suites des millénaires qui seraient nécessaires pour rendre valides, selon certains auteurs, les conclusions obtenues par le calcul des probabilités concernant les crues extraordinaires. On peut penser que, dans certains bassins, une évolution du climat local à plus courte échéance peut être prévue, résultant non pas de phénomènes naturels, mais de phénomènes humains (déboisement, déplacement des alpages, migrations, développement des cultures, ete...). Cetle évolution, qu'elle soit d'origine naturelle ou humaine, pourra dans certains cas apporter des informations non négligeables sur les crues de l'avenir.

De toute façon, voilà deux catégories d'informations qui nous semblent pouvoir être valables au moins dans certains cas. Or, il ne semble pas que ce genre d'information puisse entrer dans les schémas probabilistes tels qu'on les envisage la plupart du temps : pour le plus grand nombre des hydrologues, en effet, la probabilité d'un débit exprime sa fréquence approximative d'apparition sur une très Iongue suite d'années - et comment introduire la fréquence de phénomènes jamais observés, mais seulement réputés plus ou moins possibles?

Il y a autre chose : dans l'esprit que nous venons d'indiquer, une crue millénaire est définie comme la crue devant apparaitre en moyenne une fois en mille ans. L'expression " en moyenne » sous-entend qu'on devra considérer une longue suite de millénaires pour vérifier approximativement la théorie. Mais alors -- outre Ies changements de climats dont nous avons déjà parlé - que doit-on penser de l'application de cette théorie à un barrage dont la durée de vie sera peut-être de deux ou trois siècles? Il y a là un hiatus évident qui a été fort bien vu par M. Pardé; cela lui a donné l'occasion d'émettre une critique à la fois ironique et judicieuse des méthodes probabilistes ainsi conçues.

Nous venons de mettre en lumière un certain nombre de difficultés graves. Nous croyons qu'on peut les surmonter sans peine, dans le cadre de la statistique. Cela nécessite un élargissement des idées couramment admises. Mais pour en faire bien entendre à la fois la nécessité et la possibilité, nous devons entamer ici une digression sur le sens profond de la notion de probabilité.

\section{CALCUL DES PROBABILITÉS ET STATISTIQUES}

Tout d'abord, il nous parait indispensable de bien préciser ce qu'est pour nous le calcul des probabilités et ce qu'est la statistique.

Le calcul des probabilités est une branche des mathématiques - nous disons bien « des mathématiques » - et non pas des « mathématiques appliquées », car le calcul des probabilités doit être considéré comme une théorie purement abstraite, comme la géométrie de Hilber T ou la théorie des groupes : on appelle probabilités des êtres mathématiques attachés à des parties d'un ensemble abstrait, et obéissant à certains axiomes dans leurs combinaisons mutuelles. De ces axiomes, on déduira tout ce qu'on peut déduire : la se borne le ealcul des probabilités. Pour reprendre une expression de M. HaLphen, c'est "une construction ideale vide de tout contenu réel $\gg$. Maintenant il nous semble que les applications du calcul des probabilités peuvent à peu près se classer en deux grandes catégories : applications physiques, applications statistiques.

Les applications physiques sont caractérisées par le fait suivant : comme dans le cas de n'importe quelle théorie physique, il suffit de vérifier que les observations s'accordent avec la théorie pour pourvoir dire que la théorie est bonne. Il en est ainsi par exemple de la théoric cinétique des gaz. Cela tient à ce que les conséquences observables de la théorie (au moins dans la physique classique) concerment seulement des événements pratiquement certains..

Pour les autres catégories d'applications (et c'est le cas en hydrologie), il n'en va pas de même; ces applications constituent ce que j'appellerai 《la statistique ». Aux phénomènes observables sont liées des probabilités quelconques, et on se voit obligé de donner un « contenu 
réel » à la notion de probabilité. Rappelons en quelques mots comment se sont présentées historiquement les divergences sur ce point.

\section{L'ÉVOLUTION DE LA NOTION DE PROBABILITÉ DEPUIS LAPLACE}

Comme Pascal, pour qui le mot «probable 》 s'appliquait à toutes les incertitudes, depuis le problème des dés posé par le Chevalier DE MÉné jusqu'à l'ineertitude de l'existence de Dieu dans le célèbre pari, il semble que Laplace, puis Gavss et ses contemporains, aient concu le champ d'application du calcul des probabilités comme extrèmement large : je rappelle par exemple les applications qu'a tentées LAPLACE aux témoignages et aux décisions de la justice. Pour lui, toute proposition incertaine entre dans ce champ d'application.

Déjà, Poncarte dessine un abandon de cette position : témoin la préface de son Calcul des Probabilités sur les lois du hasard : car le concept de « hasard 》 est sans conteste beaucoup plus particulier que celui d' « incertitude ». Tout ce qui est au hasard est incertain, mais la réciproque n'est pas vraie : je ne crois pas qu'on puisse dire en aucun sens que le débarquement de Jules César en Angleterre soit "au hasard ».

Après Porncaré, et surtout depuis quelques dizaines d'années, les mathématiciens ont senti le besoin d'assurer mieux les fondements de la notion de probabilité : je souligne que cette tendance n'est pas un simple désir de philosophes, mais s'est imposée avec force dans quelques problèmes statistiques, à commencer par le problème de l'estimation.

Ces recherches ont abouti à scinder les théoriciens en deux grandes catégories : on les appelle parfois subjectivistes et objectivistes, mais ces termes sont peut-être mal choisis; je les nommerai intuitionnistes et empiristes (ou « fréquentistes », suivant l'expression des Anglais). Bien entendu, pour ces deux écoles, le calcul des probabilités est identique, d'après ce que nous avons dit tout à l'heure - les divergences résident dans le contenu concret donné à la notion de probabilite - ou, suivant l'expression consacrée, dans la théorie de la probabilité. Pour les empiristes, ce contenu concret se rattache à la notion de hasard, pour les intuitionnistes, à l'idée beaucoup plus générale d'incertitude. Notons que le hasard est en réalité un schéma construit par notre esprit; ce schéma est conforme aux fameuses «lois du hasard» - on admet, après l'échec de Von Mrsìs (théorie du collectif), que cette notion de hasard doit etre prise comme idée première : le hasard ne peut pas se ramener au certain. En tout cas, dans tout phénomène
« au hasard », on peut réaliser ou concevoir une suite d'éprenves, et les fréquences constatées pour les diverses réalisations tendent pratiquement vers des limites (loi des grands nombres). Pour les empiristes, la limite de fréquence est la seule interprétation valable de la probabilité. On trouvera un exposé cohérent de ce point de vue (si l'on excepte la théorie du collectif) dans l'ouvrage de Von Misìs : Probability, Siatistik und Wahrheit.

Je crois qu'il est assez évident que la notion d'incertain est beaucoup plus générale. Pour y rattacher la probabilité, il suffit d'admettre ce qui est assez intuitif - que les « degrés de croyance rationnelle », que nous pouvons avoir vis-à-vis de propositions incertaines, se combinent entre eux d'une facon dont le calcul des probabilités donne une bonne image. Je ne développerai pas davantage ce point, auquel est consacré l'ouvrage assez ancien (malheureusement aussi assez méconnu) de John MaynardKeynes : A treatise on Probability, ou encore le mémoire beaucoup plus récent (puisqu'il doit paraittre seulement dans les prochains mois) de M. HaLpren sur "La notion de vraisemblance. - Essais sur les fondements du calcul des probabilités et de la statistique mathématique ».

\section{VRAISEMBLANCE ET FRÉQUENCE \\ ETAT ACTUEL de LA CONTROVERSE}

Au terme de l'évolution des idées dont nous venons de domner seulement une vue très partielle, on se trouve donc devant deux possibilités, quant au contenu réel à donner à là notion de probabilité, que nous désignerons par « fréquence » et « vraisemblance ». En réalité, le concept de « vraisemblance » nous semble infiniment plus général. Mais, dans la pratique, un très grand nombre de statisticiens, particulièrement parmi les Anglo-Saxons qui ont, peut-on dire, créé la statistique, ont travaillé dans la perspective empiriste, ou «fréquentiste». On peut se demander pourquoi, et le statisticien anglais BARNaRd en donne une explication aussi sensée qu'amusante : "C'est un sujet de méditation, dit-il, de chercher pourquoi les probabilistes, depuis Laplace, ont employé les probabilités (au sens de fréquence) plutôt qu'une notion plus générale. Cela est dû à un caractère très général de la pensée scientifique du $x x^{\circ}$ siècle, qui conduisait les savants à se croire capables de concevoir l'ensemble de toutes les possibilités universelles, ou cela est peut-être dù aux habitudes sédentaires des mathématiciens, qui les conduisent à s'intéresser aux jeux de cartes et d'intérieur, lesquels peuvent habituellement être menés jusqu'à une conclusion définie, plutôt qu'aux 
sports de plein air comme les courses de chevaux, dans lesquelles les intempéries peuvent empêcher toute issue envisagée d'avance?

J'ajouterai que les problemes hydrologiques me semblent se classer plutôt avec les courses de chevaux qu'avec les jeux de cartes.

Quoi qu'il en soit, on doit constater :

1" Que la controverse est loin d'ètre close parmi les statisticiens les plus éminents;

2" Que c'est dans l'esprit « empiriste » qu'a été élaborée en majeure partie la technique statistique : theoric des intervalles de confiance et théories des tests de Neyman el Prarson. Ces théries conviennent dailleurs parfaitement à certains $t y-$ pes de problèmes, tels que cenx que pose le contrôle des fabrications. Parmi les intuitionnistes, les travaux de JEFrnexs ne semblent pas, et de loin, aussi convaincants. Seul le problème de l'estimation semble bien résolu par la formule de BAyEs, comme l'a montré M. HALPHEN dans le mémoire que nous avons cité. Quant à Fisher, il occupe une position assez ambiguë entre les deux écoles;

$3^{\circ}$ Cette prédominance des empiristes dans l'évolution de la technique statistique semble avoir marqué profondément la plupart des hydrologues. On voit mal, d'ailleurs, comment il en aurait pu etre autrement.

Peut-être me pardonnera-ton un peu maintenant la longue digression que je viens de faire en dehors de l'hydrologie, si j’ai réussi à montrer que la statistique, concue dans l'esprit plus large que j'ai appelé intuitionniste, balaie sans peine - au moins théoriquement - toutes les objections que j'ai signalées. Si l'on admet que la statistique est la science des décisions rationnelles a partir d'informations incertaines, on doit pouvoir $y$ intégrer, comme vraisemblances, les diverses informations dont nous avons vu qu'elles ne pouvaient entrer dans un schéma « fréquentiste $\gg$.

\section{IE PIROBLEME DES CRUES}

Il me reste à préciser un peu comment concevoir le probleme des crues dans cet esprit : si nous décidons aujourd'hui de construire un barrage dont la durće de vie semble devoir être disons de trois siècles, qu'est-ce qui nous intéresse au point de vue des crues? Est-ce la crue qui doit être atteinte ou dépassée $K$ fois en moyenne sur une durée de $K$ millénaires, comme l'assurent les fréquentistes? Nous croyons plutôt que la seule crue qui nous intéresse est la crue maximum qui se produira effectivement durant les trois siecles à venir. Cette crue concrete, il est plus on moins vraisemblable qu'elle atteindra telle ou telle valeur. Et c'est la répartition de cette vraisemblance, de ce « degré de croyance rationnelle », compte tenu de toutes les informations dont nous pouvons disposer, qui constitue en principe l'objet du probleme.

On voit bien, dans cet esprit, qu'il n'y a aucune difficulté logique à faire intervenir, pour supputer cette raisemblance, des éléments tels que l'évolution du climat, ou ee que nous avons appelé le «problème du déluge ».

Cependant, d'après ce que nous avons vu sur l'état actuel de la technique statistique, il est possible qu'on soit amené, même si l'on adopte les vues que nous venons d'exprimer, à employer les techniques existantes. Il faudra sans doute bien des années avant que des techniques intuitionnistes soient assez élaborées pour servir à l'usage des hydrologues. Mais il nous paraitt souhaitable de ne pas perdre de vue que ce n'est là qu'un pis-aller.

Ce que nous venons de dire n'a pas la prétention de constituer une ouve constructive : notre seule ambition était de montrer comment une certaine conception de la statistique - conception encore dans l'enfance du point de vue des techniques - - permet d'éliminer complètement certaines objections et de conslituer une trame logique pour une solution plus satisfaisante du problème.

\section{I S C USS I O N}

\footnotetext{
M. le Président signale que les deux premières parties da mémoire (classement des méthodes d'évaluation et philosophie des fondements du caleul des probabilités) sont l'objet d'un examen devant une Commission spéciale, à laquelle il convic tous les membres du Comité Technique intéressés par la question et que seule la troisième partie (application au problème de la Durance) serait à discuter pour l'instant en séance plénière.

M. Monlat donne lecture du commentaire ci-après, envoyé par M. Pardé:
}

\section{QUELQUES CONSIDÉRATIONS SUR CERTAINS ASPECTS DU CALCUL DES PROBABILITÉS}

l.e calcul des probabilités appliqué à la prévision des crues de fréquence très rare, ce qui revient en sommo a la prévision des debits maxima les plus forts à craindre se fait d'habitude d'après les phénomènes sans distinction de saison, más il peut être intéressant de prévoir dans la mesure du possible les phénomènes redou- 
tables susceptibles de survenir aux diverses epoques de l'annéc. Cette précaution s'impose particulièrement lor'sque les crues menacent de vastes régions cultivées.

On sait que mème les très grandes crues de saison froide ne sont pas dangereuses pour l'agriculture tant qu'elles ne comportent pas sur le champ d'inondation de courants susceptibles daracher la terre cultivable ou de te convir de sable et de gravier infertiles; mais en saison chaude, mème les débordements d'eau peu rapide et quasi stagnante détruisent les récoltes, après un ou deux jours de submersion, pour la plupart des plantes cultives. Voila pourquoi par exemple, la crue de juillet 1882 forte d'environ $5,50 \mathrm{~m}$ sur la Saone a Chalon et a Macon contre $6 \mathrm{~m}$ et plus pour les très grandes erues récentes et $8,05 \mathrm{~m}$ à Mâcon pour octobre-novembre 1840, a été dommageable.

A ce problème correspond le souci que j'ai toujours cu lorsque j'étudiais les rivières de rechercher tous les maxima dépassant telles ou telles hauteurs pour la plus longue période possible, en chacue mois. Mais, bien entendu, si l'évaluation par le calcul des probabilites comporte des incertitudes et des risques d'erreurs pour l'enscmble de Pannée, clle devient beaucoup plus aléatorre encore pour les saisons et les mois au cours desquels mème les petites crues sont rares; car dans ces cas, la batse de l'extrapolation est tres imparfaite et la fréquence de ees crues petites el movennes doit pouvoir differer beaucoup plus, d'un siècle à un autre siècle précédent uu suivant, que cela n'arrive pour les crues de meme hauteur, considéées pour l'ensemble de l'année. Et d'autre part, si le fait d'une concentration à peu d'intervalle de grandes crues dont l'intervalle moyen peut être présumé de 100 a 500 ans est susceptible de tromper sur l'extrapolation de la courbe, de semblables concentrations peuvent bien plus encore, me semble-t-il, exposer à des méprises pour les crues de quelques mois donnés.

Par exemple, les crues de saison chaude sur la Saône sont rates. Il semble que deux seulement aient depassé 6 in a Chalon of it Mateon en mai depuis plusiens siecles peutetre, Or, foutes les deux ont en licu ì quinze jours dintervalle en 1656. Puis si l'on prend te Rhone a Lyon, il peut eoles avoil des arues considerables en tous mois satuf peut-che du 15 mals a ta lin avril bet chcore l'avenir modificra-t-il sans doute cetle présomption qui ne tient pas assez compte du caleul des probabilités); mais il est curieux que les plus fortes erues d'août bien connues sur le fleuve à hyon aient eu lieu à un an seulement d'intervalle, à savoir $5,81 \mathrm{~m}$ a PontMorans en août 1851 et $5,75 \mathrm{~m}$ en août 1852 . Depuis lor's, on n'a pas vu en ce mois plus de $4,80 \mathrm{~m}$ ou un peu plus en août 1875 ; mais en 1852 , dix jours avant les $5,75 \mathrm{~m}$ cités plus laat, on observa environ $4,70 \mathrm{~m}$ et un mois plus tard, une crue aussi forte accompagnee d'un phénomene relativement plus puissant encore sur le Doubs a surtont sur le Rhin jusqu'à Bâle et Strasbourg.

fe signale ces rapprochements dans le temps a la fois pour leur interet propre et pour le ròle perturbatent qu'ils peuvent jouer dans le caleul des probabilités. A vai dire, si l'on avait la certitude que l'exirapolation soit bome, on pournit conclure d'apes elle que, par cxemple, les deux ertes d'août 1851 et 1852 surventes ¿ un an d'intervalle correspondent en réalité à un intervalle moyen de 200 ol 500 ans peutetre, sinon plus (contre 20 à 25 ans pent-etre pour les mémes phénomènes considérés dans l'ensemble de l'annéc. Mais comment être sûrs que la base de la courbe s'applique à 1.000 ans par exemple aussi bien qu'a 50 et que l'extrapolation n'est point susecptible de grosses erreurs? De toute façon, même en formulant ees critiques, je considère que les tentatives d'extrapolation limitees a des mois donnés doivent avoir un intérêt pratique. En tout cas, elles sont un jeu et un stimulant très intéressant pour l'esprit.

Un des aspects de ces extrapolations saisonnières auquel je songe est le suivant: j'ai jusqu'à présent admis qu'en certaines saisons, a moins de changements climatiques extraordinaires, les très grandes crues sont, non pas d'un intervalle extraordinairement rare, mais positivement impossible. Je continue à croire à cette impossibilité pour certaines rivières et certains mois daus les conditions présentes de climat. Mais pour d'autres rivières l'événement m'a montré que ces impossibilites n'existaient point. Par exemple, sans doute à la suite d'informations géographiques et hydrauliques insuffisantes, jaurais juge autrefois tout a fait impossible cue la fameuse crue de l'Orba en 1935 ait eu lieu en août. De même je jugeais bien de fortes crues possibles pour le Tarn et la Garonne inféricure en mars, mais jaurais presque parie qu'une crue aussi extrardinare d'origine méditerranéenne comme celle de 1930 n'aurait jamais pu dépasser par exemple 8 ou 9 m a Montauban, au lien de 11,30 qu'elle a atteints. Et d'autre part, je ne connais qu'une forte erue de juillet, celle de 1652 pour le Tarn à Montauban. Mème les crues de fable imporlance en ee mois sont extremement rares. Or, daprès les chroniques qui se confirment, les unes par les autres, il semble bien que la crue de juillet 1652 at eté non seulement très forte mais monstrucuse, avec maximum intermédiaire entre les niveaux déja connus de novembre 1766 et décembre 1772 et celui de mars 1930 . Il selait curieux de pouvoir évaluer la fréquence type d'une crue pareille en juillet ou même en juilletaoût. A priori et « au sentiment», il ne m'étonnerait pas que sa probabilité soit beaucoup plus faible encore que $1 / 1.000$ ou mene 1/5.000. Je signale cette anomalie at l'ingéniosite et à la curiosité des probabilistes de maintenant ou des millénaires à venir.

D'autre part, dans un ordre d'idées assez voisin de celui-ci, j’ai déjà signalé entre autres concentrations remarquables dans le temps, celle des crues géantes de l'Inn inférieur en 1598 et 1606 d'une part, puis en 1786 et 1787 d'autre part. J'ajoute qu'en 1786 il y eut en aoth deux très grandes erues dont l'une formidable et lautre pas tres inferieure; mais la crue de 1787 qui eut pour suite la seconde inondation bien connue du Danube at Vienne est bien moins remarquable par sa hauteur et pat son arrive moins d'un an et demi apè une autre du nême genre, que par sa date, à savoir la Toussaint 11 est entendu que l'Inn inferieur pent avoir de fortes crues en saison froide, et j'ai découvert, il y a bien longtemps, dans mes bavardages avee le tres regrette inspecteur général E. Anmand, que ces crues étaient très souvent contempolaines de eelles du Rhone supérieur et du Rhin à Bàle, «Covariation », selon le terme de M. Covtage, d'une smande importance chimatique. Mais, a cette épocque, j'aurais peut-chte admis que l'Inn à la Toussaint, tous les 500 ou tous les 1.000 ans en moyenne, atteigne 7 ou $8 \mathrm{~m}$, mais jamais 10,50 ou $11 \mathrm{~m}$. Un probabiliste aurait sans doute eu raison contre moi. Mais je persiste a juger possible ou mème probable que pour d'autres rivieres et d'autres mois il y a des limites supérieures bien plus basses, des possibilites d'extravagance bien plus réduites, quel que soit l'intervalle moyen cnvisage En tout cas, il me semble utile de poser la cluestion.

Et enfin, en voici une autre toujours liéc à la concentration dans le temps de certaines crues. Ici, la chose devient beaucoup plus grave, si je puis m'exprimer ainsi. Mon idée est que les fortes crues font beaucoup plus que pouvoir se suive à des intervalles infiniment plus faibles que les intervalles moyens, au cours de 1.000 . 5.000 ou 10.000 ans. Très souvent, par le fait même qu'elles ont eu lieu, il doit y avoir des probabilités plus ou moins fortes pour qu'elles se répètent, soit sur les mèmes rivières, soit dans la même région. Il se produit maintes fois certes des crues absolument isolées dans le: temps, mais les successions de phenomènes graves ì 
quelques semaines d'intervalle sont extrêmenent f'réquentes et parfois lá ressemblance entre les uns et les autres est, pourrait-on dire, presque hallucinante. Jo citerai, entre d'innombrables exemples, les très fortes crues méditerranéennes ou alpestres, de mécanisme curieusement semblable, tout au moins en partic, du basRhòne en octobre-novembre 1840 , en fin octobre $1830 \mathrm{et}$ 1841 , vers la Toussaint de 1843 et a la fin de septembre 1842, puis la célèbre série des crues méditeranéennes d'octobre 1907 avec quatre coups de force particulièremont brutaux en France dont trois domerent plus de 6,50 sur le Rhône a Beaucaire (et trois de ees phénomènes sur quatre étaient cévenols). En outre, dans la mème période, mais à d'autres dates il $y$ a eu plusieurs petites crues du mème genre et une autre formidable du Pô, puis une autre aussi grandiose que l'Ebre. Ou encore, je signalerai les deux crues cévenoles très redoutables du 20 et du 28 septembre 1846 suivies vers les 17 et 18 octobre par une crue moins violente sur les Cévennes mais beaucoup plus forte sur l'ensemble $d u$ Bas-Rhône et surtout sur la Loire où elle tourna au désastre, etc... Enfin, trois des six plus grandes crues connues de la Durance ont eu lieu du 25 octobre au 12 novembre 1886.

Il me parait évident qu'il serait tout à lait abusif de voir là un rapprochement purement aceidentel comme ce qui amène plusieurs catastrophes toutes égales, coup sur coup, comme les deux desastres des avions a Bahrein à deux jours d'intervalle, ou comme l'invasion de votre train, un jour donné, par le même type d'individus cnormes, bruyants, remuants, qui s'agitent dans le couloir durant tout le voyage et qui semblent stéréotypés. II $y$ a certainement des influences météorologiques qui tendent a produire des effets analogues coup sur coup en peu de temps, et lorsque l'atmosphère est ainsi détraquée, viciée, comme par une maladie, la production d'un phénomène grave a des chances d'être suivie par des phénomènes sérieux plus ou moins semblables. Et qui sait si, au fond, il n'y a pas autant de probabilités de pareilles successions que d'un isolement plus ou moins relatif lorsqu'une grande crue vient d'avoir lieu. Je me demande même, mais avec plus de doute, si ces rapprochements n'existent point dans les limiles de quelques annéces et non seulement dans celles de quelques semaines ou de quelques mois. C'est la fréquence de tels rapprochements qui m'inspirent cette pensée. Je me contente de la soumettre aux spécialistes du calcul des probabilités, appliqué à l'Hydrologie.

M. Christian Brad, ne pouvant assister anx réunions de la Commission spéciale, fait part de quelques-unes des réflexions qu'il a pu faire sur le problème en général, lorscu'il dirigeait le Service d'Annonce des Crues au Ministère des Travaux Publics :

1" La méthode Bacher (1) pourrait être ajoutée aux divers procédes d'évaluation des crues, car elle se base sur la notion de débit et permet non seulement de déduire avec une précision très satisfaisante l'importance de la montée d'après les hauteurs atteintes à l'amont, mais encore d'intégrer si besoin, dans certains cas, les données pluviométriques et même les prévisions météorologiques du bassin supéricur ou des amuents. Exemple : influence sur le nivean de la Seine a Paris des pluies arrosant le bassin du Grand-Morin. Celles-ci arrivent en 24 h sur Paris. Or, on annonce les niveaux à Paris trois ou quatre jours d'avance avec une précision de quelques centimètres.

$2^{n}$ L'application de la méthode Bachet à certains cours d'eau tropicaux à long parcours tel que le Niger

(1) Voir Mémoires et Trabaux, nค 2-1948, p. 152 a mème permis de prévoir les crues de ce fleuve en Nigeria six mois à l'avance. De plus l'associattion à la méthode Bacher d'une covariation de la Haute-Guinée où le Niger a sa source avec un bassiu de même latitude situé dans la basse Nigeria, a permis de faire les mèmes prévisions en se basant uniquement sur la climatologie de ce bassin. Malgré l'évaporation qui peut être très importante dans le bassin moyen (région deltä̈que du Niger), on arrive, sur le plan pratique, à des prévisions très satisfaisantes. C'est dire toute la souplesse et l'intérêt de Ia méthode Bacher.

$3^{\circ}$ On peut par ailleurs envisager, pour un avenir plus ou moins rapproché, l'utilisation de certaines prévisions météorologiques à longue échéance, actuellement à l'étude notamment à Dakar, sur la base de certaines périodicités; l'association de ces prévisions à la méthode Bachet peut permettre d'espérer l'évaluation des crues à longue échéance.

M. le Président remercie M. BEAU et dit tout le bien qu'il pense de la méthode Bacher pour la prévision des crues d'après l'état initial de la rivière et sa montée a l'amont. Il propose à M. BEAU de rester en contact avee M. Monlat.

En ce qui concerne les prévisions des précipitations ì longue échéance, M. Sanson indique, sur la demande de M. le Président, qu'il n'a pas entendu parler de prévisions établies sur des bases seientifiques qui puissent donner avec suffisamment de certitude des indications su" le temps qu'il fera plus d'un mois à l'avance aussi bien en France qu'à l'étranger.

A propos de la note de M. Pandé, M. Sanson confirme que les années de grandes précipitations, d'une part, ef ies années de sécheresse, d'autre part, paraissent souvent se réunir par « paquets » ou périodes. Exemple : années sèches de 1942 à 1949 inclus, coupées cependant par un semestre très pluvieux en 1944). Il ajoute, sur la demande de M. Barrillon, qu'il ne connaît pas de « binomages » dans les phénomènes météorologiques autres que les averses; l'étude de ces cas est d'ailleurs rendue difficile par le manque de préeision des observations effectuées avant 1870. (Voir cependant la note sur les anomalies du climat parisien du xur au xvar ${ }^{e}$ siècle parue dans la Météorologie de mai-juin 1937.)

M. le Président remarque que les hauteurs de crue du XVII $^{*}$ siècle eitées par M. PARdé ont pu être amplifiées par des ponts de débouché insuffisant ou divers obstacles encombrant les rivières tels que moulins, pecheries, etc.

En ce qui concerne l'application particulière à la Durance, M. le Président demande à M. Mortat si les résultats pourront être connus assez tôt póur évaluer les crues au barrage de Serre-Ponçon, dont la construction est prévue dans un délai assez rapide; l'importance de ce barrage qui sera le plus haut d'Europe en matériaux compactés (110 $\mathrm{m}$ de hauteur, cuvette de un milliard de $\mathrm{m}^{3}$ ) et sa nature excluant la possibilité de submersions exigent des évacuateurs de crues largement conditionnés.

M. MonLat répond que des renseignements déjà appréciables pourront etre obtenus au bout d'un petit nombre d'années d'observations : il n'est pas douteux que ces renseignements pourront être d'un certain prix dans le choix de dimensions des évacuateurs de Serre-Ponçon.

M. Lugrez ajoute quelques remarques qui seront utiles a M. Monfat pour l'étude de la Durance:

10 Outre les pluviomètres de la vallée et celui du Lautaret, il existe une station bien située à SaintVéran $(2.200 \mathrm{~m})$ et aussi un certain nombre de nivopluviomètres totalisateurs. 
2. Anticipant sur le vou formule par M. Moriat', et relatif à la mesure de la couche de neige au sol, le Service de la Production Hydraulique d'E.D.F. possède des mesures faites chaque semaine depuis deux ans en trois postes.

$3^{\prime}$ Il serait souhaitable de répartir les stations pluviométriques sur des régions d'exposition différente, mais il $y$ a à cela une difficulté car les lieux habités sont situés principalement sur les versants ensoleillés.

4" Il conviendrait de multiplier les stations de mesure d'insolation, dont l'intérèt n'a certainement pas échappé à M. Monlat.

5 " Les hydrogrammes élémentaires dont le tracé préalable est nécessaire pour l'élaboration d'hydrogrammes synthétiques devmient être ètablis pour des parties de bassin d'orientation différente.

M. le Président remercie M. Lugiez.
Sur la demande de M. le Président, M. Crosner indique que, pour l'Algérie, les problèmes qui se posent rentrent dans le cadre des exposés qui ont éle faits.

M. Rabrguer estime que les études doivent porter sur les causes du phénomène en mème temps que sur le phénomène luímême. L'Energie Electrique du Maroc poursuit actuellement des etudes sur les types de temps et leur sucession chronologique en mence temps que sur les débits. Ces études devaient permettre de déterminer s'il existe des variations eycliques des types de temps et si une corrélation peut etre établie entre les pluies et les débits.

M. Sanson signale qu'il y a deux services météorologiques au Maroc: le S.MM.A. (Service Météorologique de la Métropole et de l'Afrique du Nordi) et l'office Scientifique Chérifien, ce dernier s'occupant plutot des questions de climatologie. La collaboration des deux services est dejà éiroite mais un projet à l'étude la rendrait encore plus complète.

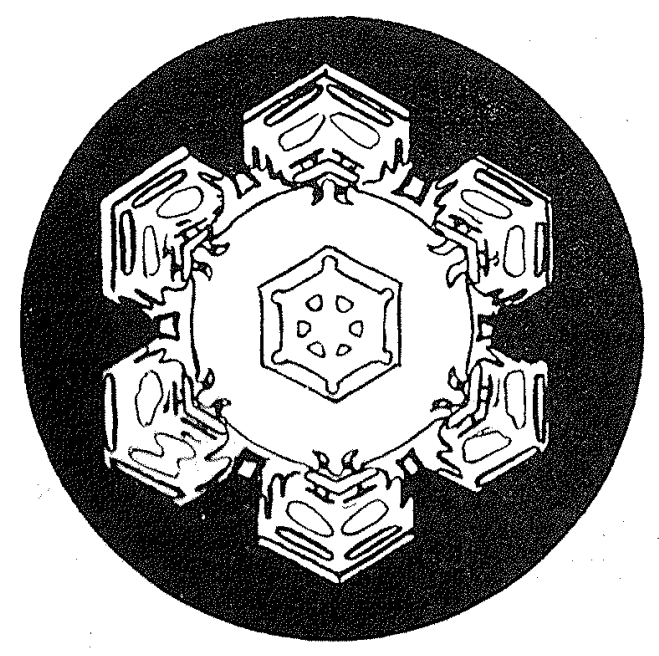

